\title{
Evaluación de las técnicas de aislamiento y contención por parte de los equipos de enfermería en los hospitales psiquiátricos griegos
}

C. Lemonidou, $\mathrm{RN}, \mathrm{MSc}, \mathrm{PhD}^{*}$

M. Priami, RN, PhD*

A. Merkouris, $\mathrm{RN}, \mathrm{MSc}, \mathrm{PhD}^{* *}$

M. Kalafati, $\mathrm{RN}, \mathrm{PhD}^{* * *}$

C. Tafas, $\mathrm{RN}, \mathrm{MSc}^{\star * * *}$

C. Plati, RN, PhD*

* Universidad de Atenas. Escuela de Enfermería

** Evangelismos Hospital

*** Hospital de KAT

GRECIA

**** Universidad de Connecticut USA

RESUMEN - Objetivo: a) Investigar el tipo de restricción utilizada con el fin de eliminar el comportamiento violento de pacientes psiquiátricos,b) Explorar las actitudes del personal de enfermería frente al aislamiento y la contención y c) Determinar si hay alguna diferencia en las capacidades del personal de enfermería debido a su nivel educativo y a los años de experiencia.

Método: La muestra estaba formada por 190 enfermeros/as de doce unidades psiquiátricas correspondientes a cinco hospitales diferentes en donde se distribuyó un cuestionario auto-administrado y se realizó un análisis estadístico descriptivo junto con la aplicación del test chi cuadrado.

Resultados: Las restricciones realizadas con mayor frecuencia incluían las contenciones corporales (42,6\%), el aislamiento en la propia habitación (26\%), y ambas $(31,1 \%)$. El aislamiento o la contención se utilizan en la mayoría de los casos para la seguridad del paciente $(70,5 \%)$, el control de su comportamiento $(23,2 \%)$, y para comodidad del personal $(0,5 \%)$. El ochenta por ciento de los enfermeros/as está a favor de la contención mientras que un $16,8 \%$ está en contra. No hubo diferencias estadísticamente significativas en las actitudes de los enfermeros/as motivadas por su nivel de formación y experiencia laboral. 


\section{Introducción}

El aislamiento es una de las intervenciones más antiguas que todavía persiste en el tratamiento de personas con enfermedades mentales según ya describió Soranus, filósofo griego del siglo II d. C. (McCoy y Garritson 1983). La responsabilidad de crear y mantener un entorno seguro para los pacientes y para el personal tradicionalmente ha pertenecido a los enfermeros/as psiquiátricos (Cahill, Stuart, Laraia y Arana 1991, Sclafani 1986) especialmente en urgencias y en hospitalización, situaciones en las que se precisa una actuación rápida con el fin de predecir y controlar la violencia potencial (Anderson y Roper 1991,Grey y Diers, 1992, Morrison 1993). El aislamiento se ha empleado en un esfuerzo por lograr estos objetivos de la enfermería y se percibe por algunos de ellos como una estrategia terapéutica y eficaz para controlar situaciones violentas (Gutheil y Daly 1980, Chu y Ryan 1987). Su pertinencia, no obstante, es discutible. Las filosofías contemporáneas de la enfermería en salud mental muy dadas al humanismo, al individualismo y a la relación terapéutica enfermero-paciente, muestran una sensibilidad opuesta del aislamiento como representante todavía de una actividad de enfermería anticuada que no tiene cabida dentro de las modalidades de tratamiento actuales (Sallah 1992, Topping Morris 1992).

Además, los poderosos argumentos legales y morales y la controversia en cuanto a la eficacia terapéutica de la utilización del aislamiento y la contención, están invitando a los profesionales de la salud mental a examinar de forma crítica su papel en el cuidado de personas con enfermedades mentales (Brown y Tooke 1992, Soloff 1987). Alty y Mason (1994) comentan que mientras que el tratamiento humano se constituya como el enfoque principal del cuidado, la utilización de estas estrategias sirve como un eficaz medio de control, apoyado en parte por un clima de atención custodial y por una profesión médica desalentada. Otros han sugerido que el control que ofrece el aislamiento permite al personal mantener niveles elevados de eficacia, al facilitar un entorno conformista, reduciendo la ansiedad producida por el trabajo en la institución psiquiátrica (Menzies 1970, Brown 1973, Beardshaw 1981). Esta posición se apoya en los trabajos de Morrisson (1989, 1993) y Rosenhan (1973), que han descrito cómo el personal destaca el valor del control dentro de un modelo de atención estrictamente autoritario.

En Grecia, recientemente los enfermeros/as han empezado a investigar los problemas a los que se enfrentan en los entornos psiquiátricos, tras el establecimiento de esta formación universitaria en el nivel de licenciatura y doctorado. Los únicos datos disponibles están en relación con pacientes en las Unidades de Agudos (Plati et al. 1995) y respecto al efecto de la hospitalización involuntaria sobre la sintomatología clínica de los pacientes psiquiátricos a diferencia de lo que sucede con los pacientes hospitalizados voluntariamente (Mougia 1999). La falta de datos suficientes indica la necesidad de que se realicen investigaciones más exhaustivas.

La finalidad de este estudio es: a) Investigar el tipo de restricción utilizado para controlar el comportamiento violento de pacientes psiquiátricos, b) Explorar las actitudes del personal de enfermería hacia el aislamiento y la contención y c) Determinar si hay alguna diferencia en cuanto a las actitudes del personal de enfermería motivada por su nivel educativo y sus años de experiencia. 


\section{Revisión de la literatura}

Muchos autores definen el aislamiento en términos equívocos como si no fuera necesario que sea evidente (Binder 1979, Schwab y Lahmeyer 1979, Soloff y Turner 1981, Hodgkinson 1985). No obstante, se ha definido como "...la reclusión forzada de una persona durante un periodo de tiempo arbitrario, en una habitación construida a propósito, con pocos elementos de mobiliario excepto un colchón y una cama". (Thorpe 1980, Baradell 1985).

El aislamiento se considera preferible a la medicación y a la terapia electroconvulsiva porque causa menos efectos colaterales, a pesar del riesgo potencial de su mala utilización. La base teórica de su empleo en pacientes psiquiátricos hospitalizados se apoya en los principios de contención,aislamiento y reducción sensorial (Gutheil 1978). La contención sirve para limitar el entorno del paciente mientras que el aislamiento pretende ofrecer un paréntesis al dolor y a la dificultad de relación sobrecargada del enfermo. Por otro lado, ofrece un entorno tranquilo y monótono reduciendo la actividad sensorial que puede ser insoportable en pacientes que son vulnerables a las distorsiones perceptivas y que no pueden construir sus propias barreras sensoperceptivas. Esto puede ser beneficioso en pacientes que sufren de un episodio de psicosis hiperestésica por ejemplo. (Kennedy, Williams y Pesut 1994). Como defendía Redmond (1980) el aislamiento debería utilizarse sólo como un último recurso, y siempre que ello implique una intervención tanto de gestión como terapéutica.

Investigaciones previas son ambiguas en cuanto a la utilización del aislamiento como tratamiento complementario en el cuidado de individuos con trastornos agudos o como mecanismo de control de su comportamiento (Plutchik y Larasu 1978, Campbell et al. 1982, McCoy y Garritson 1983). La realidad es que hasta la fecha la razón terapéutica del aislamiento no ha sido demostrada.

A pesar de ello, los miembros del personal cuidador creen que el aislamiento es muy beneficioso en cuanto a la modificación de comportamientos indeseables y con el fin de recuperar el control emocional del paciente (Plutchik et al. 1978). En este estudio, cuando se preguntó a los profesionales sanitarios quién se beneficia más de la experiencia de aislamiento (el personal, los otros pacientes o los propios pacientes aislados), resultó que los enfermeros/as, más que cualquier otro profesional,creían que era el paciente recluido el más beneficiado. Una década más tarde, en un estudio realizado por Cangas y Shopflocher (1989), se vio que los enfermeros/as persistían en sus actitudes positivas hacia la utilización del aislamiento y creían firmemente en su eficacia. En este estudio se dieron algunas variaciones en su utilización, lo cual puede atribuirse a las características propias del entorno que estaban parcialmente bajo control administrativo.

La literatura revela que existe controversia sobre las razones que pueden llevar al aislamiento de los pacientes. La razón principal argumentada por el personal para aislar a un paciente suele ser una agitación creciente y no necesariamente un episodio violento (Oldhan, Russakoff y Prusnofsky 1983). Un estudio de Hopkins y Lowery (1994) comparaba las causas para el aislamiento y la contención según lo que pensaban los pacientes y los enfermeros/as. Los enfermeros/as estaban de acuerdo unánimemente en que el aislamiento y la restricción están en relación con características particulares de cada uno de los pacientes. La decisión de enfermería de aislar a un paciente no estaba influenciada ni por el entorno ni por los encuentros previos. Los 
pacientes, por el contrario, estaban divididos entre la causalidad interna y externa utilizada en este estudio. Los enfermeros/as en un estudio presentado por Babrick (1981) se mostraban también reacios al hecho de que las condiciones ambientales o de situación determinaran el aislamiento y restricción de los pacientes, lo que podría reflejar la ambivalencia a menudo exhibida por la enfermería sobre el empleo de estas medidas (Kilgalen 1977, Wadeson y Carpenter 1976).

Existe una evidencia empírica que sugiere que el entorno de atención puede influir sobre la tendencia de un paciente a actuar de una forma violenta, lo que puede precipitar el empleo del aislamiento y la contención (Babrick 1981). Además se observó que las actitudes del personal, especialmente aquellas "demasiado autoritarias", contribuían a la aparición de comportamientos violentos en los pacientes del estudio de Braisford y Stevenson (1973) sobre el control de comportamiento violentos e impredecibles en los hospitales psiquiátricos. Otros autores sin embargo sostienen que las evidencias que sugieren que los rasgos ambientales y del personal influyen en la utilización del aislamiento, no son concluyentes (Lanza, Kayne, Hicks y Milner 1991, Outlaw y Lowery 1992).

\section{La contención}

En 1978, tanto el aislamiento como la contención fueron descritas por Gunderson como un componente necesario en el entorno terapéutico de pacientes ingresados, a pesar de su naturaleza controvertida. Se desarrollaron varios criterios para legitimar el empleo de la contención física incluyendo la advertencia de que no debía ser más violenta o forzada que el acto inicial de la persona trastornada, y siempre en relación con la resistencia ofrecida por el paciente.
Las fijaciones también deberían ser retiradas cuando ya no se haga probable una amenaza real de daño para el propio paciente, las personas de su entorno o para la propiedad (Rawls 1972). De hecho, siempre y cuando la contención de una persona con graves trastornos no conlleve un tratamiento degradante o inhumano, según establece la Declaración Universal de los Derechos Humanos (Asamblea General de las Naciones Unidas 1948), se considera legítima.

Los trabajos publicados anteriormente han apoyado el empleo apropiado de la contención física en lo que respecta al control de personas con trastornos graves. Rosen y Di Giacomo (1978), señalaron a los comportamientos violentos por parte de los pacientes como la indicación principal para la utilización de la contención, no obstante, la bibliografía actual sobre su empleo en enfermería psiquiátrica es escasa (Ackner 1964, Burr y Budge 1976, Darcy 1984). Las técnicas aceptadas sobre la contención física y en qué circunstancias podría ser justificable su empleo, son el punto principal de varias de las publicaciones más recientes (Stilling 1992, Tarbuck 1992,Colegio Real de Enfermería 1992).

Aunque la utilización de la contención puede llegar a crear conflicto para algunos enfermeros/as que trabajan en unidades psiquiátricas (Di Fabrio 1981), el personal de enfermería del estudio de Hopkins y Lowery (1994) unánimamente $(98,8 \%)$ declaró que la restricción era la intervención apropiada en determinados comportamientos. Y resulta llamativo que sólo el 34,5\% de los pacientes creían que su comportamiento les llevaría a la restricción.

Los datos de una investigación en hospitales de agudos en Grecia, indican que la mayor parte de los enfermeros/as (62\%) creen que la contención es necesaria como medida de protección en el caso de pacientes 
problemáticos, evitando por ejemplo lesiones (67\%), y en otros casos manteniendo adecuadamente las vías intravenosas $(76,1 \%)$. Otras razones adicionales incluían: confusión $(75,6 \%)$, seguridad de los pacientes $(67 \%)$, autolesiones $(66,5 \%)$, y el mantenimiento de los sondas urinarias $(65,8 \%)$ y tubos nasogástricos $(58,9 \%)$. Las barandillas laterales se utilizaban con más frecuencia en pacientes de alto potencial de riesgo de lesionarse, con alteraciones del pensamiento o con movilidad alterada. Los efectos de la contención en los pacientes eran: agresividad hacia el personal $(61,5 \%)$, hostilidad $(50,5 \%)$, alteración de la comunicación $(26,6 \%)$, aislamiento social $(15,8 \%)$, y negación a alimentarse (12,2\%) (Plati et al. 1995). No encontramos otros estudios con datos comparables sobre el empleo de la restricción física en hospitales generales o psiquiátricos griegos o en instituciones de larga estancia.

\section{Material y método}

Muestra: La muestra estaba formada por 190 enfermeros/as (85 titulados y 105 con dos años de formación en una escuela profesional de enfermería) que trabajaban en Unidades psiquiátricas. El estudio fue realizado en doce Unidades psiquiátricas de cinco hospitales: dos hospitales psiquiátricos con un total de nueve Unidades y tres hospitales generales con un total de tres. En los hospitales psiquiátricos, siete Unidades eran abiertas y dos cerradas y las camas de los pacientes en cada sala oscilaban entre 30 y 80 , mientras que en los hospitales generales las tres Unidades eran abiertas con 20 pacientes cada una. Los hospitales psiquiátricos ofrecían atención a largo plazo frente a las Unidades de Psiquiatría de los hospitales generales en los que la estancia media era de tres meses.
Todas las Unidades psiquiátricas atendían a pacientes con esquizofrenia, trastornos del humor, ansiedad, trastornos de personalidad, anorexia nerviosa y otros trastornos.

La Dirección y el Comité Científico de cada uno de los hospitales autorizaron el estudio. Todos los enfermeros/as aceptaron participar en el mismo, utilizándose un breve resumen en el que se explicó el significado y la finalidad del estudio, garantizando el anonimato y recogiéndose el consentimiento informado.

Instrumento: Se desarrolló un cuestionario auto-administrado para recoger los datos sobre actitudes de los enfermeros/as frente al aislamiento y la contención en los pacientes psiquiátricos. La definición de "aislamiento" dada por Thorpe (1980) y Baradell (1985) fue adoptada para este estudio, mientras que la "contención"incluía la restricción física usando para ello, por ejemplo, pulseras o correas para una mano, para las dos, correas para los pies y barandillas laterales. El cuestionario fue diseñado con las aportaciones de expertos (un profesor de Psiquiatría, dos enfermeros/as con especialidad en Psiquiatría, y dos enfermeros/as expertos en salud mental), enfermeros/as, pacientes y todo ello tras una amplia revisión de la literatura. La validez del contenido fue establecida por un panel de expertos. La herramienta fue puesta a prueba con anterioridad a este estudio por quince enfermeros/as utilizando una muestra aleatoria. Finalmente se realizaron pequeños cambios para simplificar la redacción de las preguntas. La escala de actitudes consistía en 10 preguntas cerradas. Dos preguntas se referían a la frecuencia de su utilización, de acuerdo con el tipo de confinamiento y los turnos; tres correspondían a la eficacia y a los sentimientos de los pacientes confinados; dos eran relativas a la prevención del confinamiento y finalmente tres preguntas se utilizaban para explorar los sentimientos del personal en rela- 
ción con la finalidad y la pertinencia del uso del aislamiento y la contención. La coherencia interna del cuestionario fue comprobada utilizando el coeficiente alfa de Crombach, que era de un 0,83 . El cuestionario se contestaba en unos 10 minutos aproximadamente.

Análisis: Se realizó un análisis estadístico utilizando la estadística descriptiva y el test de chi cuadrado.

\section{Resultados}

De los 190 enfermeros/as (60 hombres y 130 mujeres) el 35\% tenían una experiencia laboral que oscilaba de 6 a 10 años y que en un $30 \%$ era de más de 10 años. La mayoría eran auxiliares de enfermería (55,3\%), mientras que el $44,7 \%$ eran titulados.

Las limitaciones más frecuentes incluían contención corporal $(42,6 \%)$, aislamiento en su habitación $(26 \%)$ y ambas $(31,1 \%)$. Ante la elección, el $69 \%$ de los enfermeros/as preferían el aislamiento frente a la contención. De acuerdo con el $51 \%$ de los enfermeros/as, la contención se utilizaba con mayor frecuencia durante el turno de tarde.

Los enfermeros/as declararon que las respuestas más frecuentes de los pacientes a la utilización de la contención eran la agresividad $(76,9 \%)$, la ira $(59 \%)$ y la tensión $(51,6 \%)$. Raramente respondían con tranquilidad
(20,6\%) o regresión (17,3\%). Según los enfermeros/as, el $55,8 \%$ de los pacientes no repetían el comportamiento agresivo y el $42,6 \%$ tras retirarse la restricción. También señalaron que, en su opinión, el método más eficaz es el aislamiento en la habitación (52,6\%) comparado con la contención corporal $(33,7 \%)$.

Los enfermeros/as creen que la evaluación de los pacientes $(53,7 \%)$ y la comunicación frecuente $(32,6 \%)$ son las prácticas más importantes para evitar el comportamiento violento. El personal se reconocía como el factor ambiental más importante $(56,3 \%)$ que influye sobre la utilización del aislamiento o la contención física, siendo el nivel educativo de los enfermeros/as $(38,9 \%)$ relativamente bueno.

La tabla I describe los sentimientos de los enfermeros/as cuando utilizan el aislamiento o la contención. La mayoría de los enfermeros/as $(41,6 \%)$ muy a menudo sienten simpatía hacía el paciente y nunca satisfacción con el cumplimiento de su deber $(43,2 \%)$. La tabla II muestra su opinión sobre la razón principal para la contención de los pacientes. El aislamiento o la contención se utilizan en la mayoría de los casos para la propia seguridad de los pacientes $(70,5 \%)$, el control de su comportamiento $(23,2 \%)$ y la comodidad del personal $(0,5 \%)$. Finalmente, a pesar de los sentimientos ambivalentes expresados por los enfermeros/as, el $80 \%$ están a favor, frente a un $16,8 \%$ que está en contra.

Tabla I

Sentimientos de los enfermeros/as al utilizar medidas de contención

\begin{tabular}{lcccc} 
Sentimientos & Nunca & A veces & A menudo & Muy a menudo \\
\hline Culpabilización & $42(22,1 \%)$ & $88(46,3 \%)$ & $41(21,6 \%)$ & $19(10 \%)$ \\
Desacuerdo & $21(11,1 \%)$ & $60(31,6 \%)$ & $70(36,8 \%)$ & $39(20,5 \%)$ \\
Simpatía & $22(11,6 \%)$ & $35(18,4 \%)$ & $54(28,4 \%)$ & $79(41,6 \%)$ \\
Satisfacción por el deber cumplido & $82(43,2 \%)$ & $73(38,4 \%)$ & $23(12,1 \%)$ & $12(6,3 \%)$ \\
\hline
\end{tabular}


Tabla II

Razones de los enfermeros/as para la restricción de los pacientes

\begin{tabular}{lcccc} 
Rationale & Nunca & A veces & A menudo & Muy a menudo \\
\hline Control de la conducta & $27(14,2 \%)$ & $54(28,4 \%)$ & $65(34,2 \%)$ & $44(23,2 \%)$ \\
Seguridad del paciente y de los otros & $2(1,1 \%)$ & $15(7,9 \%)$ & $39(20,5 \%)$ & $134(70,5 \%)$ \\
Conveniencia del personal & $133(70 \%)$ & $46(242 \%)$ & $10(5,3 \%)$ & $1(0,5 \%)$ \\
\hline
\end{tabular}

El test chi cuadrado demostró que no existían diferencias estadísticamente significativas en cuanto a las actitudes de los enfermeros/as con respecto a la contención, motivadas por su nivel formativo, su experiencia en el trabajo o el tipo de hospital, excepto para la cuestión sobre el factor más importante que era el de evitar la contención de los pacientes $(\mathrm{p}<, 01)$. La mayoría de los enfermeros/as titulados $(57 \%)$ creían que la formación era el factor más importante para evitar la restricción de los pacientes seguido de la dotación de personal (43\%), mientras que la mayor parte de los auxiliares de enfermería $(71,6 \%)$ creían que la dotación de personal era el factor más importante y sólo el $28,4 \%$ citaban la formación.

\section{Discusión}

El objetivo del estudio no era describir las dimensiones éticas del utilización del aislamiento o de la contención física sino, más bien, revelar el tipo de contención realizada por los enfermeros/as y sus actitudes hacia su empleo. El tipo de restricción más frecuente utilizado en las Unidades psiquiátricas era la contención corporal y el aislamiento en la habitación, con preferencia hacia el aislamiento. Ello podría significar que los enfermeros/as se sienten más cómo- dos cuando no han de imponer la fijación mecánica a los pacientes.

El hallazgo más concluyente de este estudio es que las actitudes de los enfermeros/as respecto del aislamiento son muy positivas, pero está claro que el descriptor principal de esta actitud positiva es necesario, pero no "deseable". El ochenta por ciento de los enfermeros/as declararon que estaban a favor del aislamiento y sólo el 16,8\% en contra. El aislamiento puede verse como necesario, tras una provocación repetida por parte del paciente, y no como medio de coacción o como medida para aliviar la carga laboral de la enfermería. Esto es coherente con los hallazgos del estudio en las Unidades de agudos de hospitales griegos (Plati et al. 1995). Los enfermeros/as creen que la contención es beneficiosa en lo que respecta a la modificación de los comportamientos indeseables, como se observó en otros estudios (Wadeson y Carpenter 1976, Kilgalen 1977, Plutchnik et al. 1978, de Cangas 1993).

Las razones que esgrimen para utilizar la contención con los pacientes, como se vio en otros estudios, son la propia seguridad de los enfermos $(70,5 \%)$, el control de sus comportamientos $(23,2 \%)$ y en un porcentaje muy pequeño de los mismos $(0,5 \%)$ la comodidad del personal. En los años sesenta, Plutchik y colaboradores (1978) vieron como el personal consideraba el empleo del aisla- 
miento como muy beneficioso en lo que respecta a la modificación de comportamientos indeseables, así como para recuperar el control emocional del paciente. Tardiff y Mattson (1984) y Fassler y Cotton (1992) señalaban que la mayoría de los encuestados citaban al peligro para ellos mismos y para los demás como la indicación principal. En el estudio de Muir-Cochrane y Adult (1996) el control venía a ser la razón principal para el utilización del aislamiento y observaron que la seguridad de los pacientes y del personal, era la razón predominante en las Unidades psiquiátricas estudiadas. Los internistas señalaban que la finalidad del aislamiento era contener a un individuo, mantener la seguridad, disminuir los estímulos a los que estaba expuesto y ofrecerle una oportunidad para recuperar el control. Todos estos hallazgos indican que la seguridad y el control de los comportamientos indeseables del paciente son las razones principales para el empleo de estas técnicas.

Aunque el aislamiento y la contención pueden llegar a impedir lesiones, disminuyendo la agitación, se ha establecido que estos procedimientos pueden también llegar a tener efectos perjudiciales serios tanto físicos como psíquicos, en los pacientes. Los efectos negativos de la propia restricción en lo que respecta a los sentimientos de los pacientes de este estudio,fueron la agresividad, la ira y el estrés, similares a los hallazgos encontrados en los hospitales de agudos en Grecia (Plati et al. 1995). Binder y McCoy y Garritson (1983) detectaron que estos expresaban fuertes sentimientos negativos sobre la experiencia del aislamiento. Plutchik et al. (1978) investigaron las percepciones de pacientes aislados y hallaron que los pacientes que habían sido aislados, se sentían molestos cuando otros eran aislados y ellos mismos se sentían aburridos y enojados mientras se hallaban en situación de aislamiento. No obstante, se afirmaba que el aislamiento ayudaba a que se calmaran. En este estudio,ellos "raramente" señalaron efectos de relajación $(20,6 \%)$ o de regresión $(17,3 \%)$ en estos pacientes. De hecho, los pacientes a menudo comunicaban niveles mayores de ansiedad, ira, depresión, humillación, abandono, soledad y pérdida de dignidad después de un confinamiento (Strumpf y Evans 1988).

Aunque los enfermeros/as expresaron una actitud positiva respecto al aislamiento y a la restricción, los resultados demuestran que sienten cierto conflicto interno acerca de su utilización, ya que informaron sobre sus sentimientos con un grado significativo de culpa, decepción, simpatía e insatisfacción. La mayoría de los enfermeros/as con mucha frecuencia sentían simpatía por el paciente $(41,6 \%)$ y nunca sentían satisfacción por el cumplimiento de su cometido $(43,2 \%)$. Esto puede querer decir que los enfermeros/as, aunque comuniquen una actitud positiva respecto a la contención de los pacientes, la emplean sólo para el beneficio del paciente y no para reducir la carga laboral. Los resultados de otros estudios son similares (Plutchik et al. 1978, Lion 1987, Craig, Ray y Hix 1989).

Una serie de trabajos de investigación han mostrado cómo el número de empleados de la unidad y la hora del día podían influir también en la decisión de aislar y restringir a un paciente (Gerlock y Solomons 1983, Oldham et al. 1983, Soloff y Turner 1981). De Congas (1993) señalaba diferencias entre los turnos en el empleo del aislamiento, mientras que en otros estudios no se encontraron diferencias significativas (Mattson y Sacks 1978, Plutchik et al. 1978, Schwab y Lahmeye 1979). De acuerdo con los enfermeros/as (97 de 190, 51\%), en nuestro estudio, la contención se utiliza con más frecuencia durante el turno de tarde. Esto concuerda 
con los hallazgos de Mougia (1999) que indican que el 46,2\% (tamaño de la muestra 997 pacientes) de los pacientes estaban en situación de contención durante el turno de tarde, el 5,9\% durante el turno de la mañana y el $16,0 \%$ durante el turno de la noche. Roper et al. (1985) hallaron como punto más alto de la contención las mañanas, no las tardes, además de que la medicación no reducía de forma significativa el tiempo pasado en situación de confinamiento. Esta diferencia en cuanto a los turnos puede deberse al número de personal presente. En muchos hospitales psiquiátricos griegos, por ejemplo, los turnos de tarde y de noche pueden tener una dotación de personal de dos o tres enfermeros/as atendiendo a 60-80 pacientes, mientras que en el turno de mañana la dotación puede ser de cinco o seis enfermeros/as. Además, en los hospitales psiquiátricos la mayor parte de los pacientes están abandonados por su familia y dependen totalmente del personal de enfermería.

Con respecto a la prevención del comportamiento violento, los enfermeros/as creen que la valoración de los pacientes $(53,7 \%)$ y la comunicación frecuente con ellos $(32,6 \%)$ son las prácticas más importantes. Algunos autores señalan que el cambio debe partir de los enfermeros/as, que son los que tienen mayor presencia entre los pacientes (Roper, Coutts, Sather y Taylor 1985). En los hospitales de agudos griegos, la presencia mayor de familiares es una práctica común, pero este no es el caso en hospitales psiquiátricos porque la propia política de los hospitales no lo permite, exceptuando las horas de visita específicas que no pueden sobrepasar las tres horas.

Mientras que Mattson y Sacks (1978) sugieren que la experiencia del personal puede desempeñar un papel en el empleo del aislamiento, Schwab y Lahmeye (1979) no encuentran correlación alguna entre la experiencia de la enfermería psiquiátrica y la frecuencia del empleo del aislamiento. En el presente estudio no hubo diferencias estadísticamente significativas en cuanto a las actitudes de los enfermeros/as hacia el aislamiento o la contención motivadas por su nivel formativo y experiencia profesional, excepto para la cuestión sobre "el factor más importante para evitar la contención de los pacientes" ( $\mathrm{p}<, 01)$. La mayoría de los enfermeros/as titulados/as (57\%) creían que la formación era el factor más importante para evitar la restricción de los pacientes, seguido por la dotación de personal (43\%), mientras que la mayor parte de los auxiliares de enfermería $(71,6 \%)$ creían que la dotación de personal era el factor más importante. Esto último es coherente con los hallazgos de Cangas (1993) que descubrió que las condiciones de la Unidad tenían influencia en el empleo del aislamiento y que el número de horas de aislamiento en una unidad aumenta conforme el número relativo de personal se reduce. En 1980 en Grecia, la atención de pacientes psiquiátricos estaba basada en nueve hospitales psiquiátricos públicos sobrecargados, de los cuales muchos eran de tipo asilar y tenían un personal inadecuado, y además había una serie de hospitales psiquiátricos privados (Madianos 1994). A lo largo de los últimos 11 años se han observado cambios profundos en el sistema de atención en salud mental que hacen hincapié en la descentralización de la atención y en el establecimiento de departamentos psiquiátricos en los hospitales generales (Madianos et al. 1999). La cuestión es si estos cambios tienen influencia en los modos de tratamiento de los pacientes internados y en la utilización anacrónica de la contención. Se necesita realizar más investigaciones en los hospitales psiquiátricos griegos para descubrir los factores que conducen a una menor utilización del aislamiento y la contención. 


\section{Bibliografía}

ACKNER, B. Handbook for Psychiatric Nurses. Bailliere Tindall \& Cassell, London, 1964.

ALTY, A., MASON, T. Seclusion: A Break with the Past. Chapman and Hall, London, 1994.

ANDERSON, N.L.R., ROPER, J.M. The interactional dynamics of violence, Part II: Juvenile detention. Archives of Psychiatric Nursing, 5, 216-222, 1991.

BABRICK, K. Assessing patient violence in the health care setting. Western Interstate Commission for Higher Education, Report on Violence, pp 1-113. Colorado: Wiche, 1981.

BARADELL, J. Humanistic care of the patient in seclusion. Journal of Psychosocial and Mental Health Services 23(2), 9-14, 1985.

BEARDSHAW, V. Conscientious Objectors at Work, Mental Hospital Nurses: A Case Study. Social Audit,London, 1981.

BINDER,R.L. The use of seclusion on an in-patient crisis intervention unit. Hospital \& Community Psychiatry 30(4), 266-269, 1979.

BRAISFORD, D., STEVENSON, J. Factors related to violent and unpredictable behaviour in psychiatric hospitals. Nursing Times, 69(3), 9-11, 1973.

BROWN, G. The mental hospital as an institution. Journal of Social Science and Medicine 7, 407-424, 1973.

BROWN, J.S., TOOKE, S.K. On the seclusion of psychiatric patients. Social Science in Medicine, 35,711-721, 1992.

BURR, J., BUDGE, U.V. Nursing the Psychiatric Patient. Bailliere Tindall, London, 1976.

CAHILL, C.D., STUART, G.W., LARAIA, M.T., ARANA, G.W. Inpatient management of violent behaviour:Nursing prevention and intervention. Issues in Men tal Health Nursing, 12, 239-252, 1991.

CAMPBEL, W., Shepherd H. \& Falconer F. The use of seclusion Nursing Times 78(430), 1982.

CHU, C.C., RYAN, S.J. The role of seclusion in psychiatric hospital practice. The Psychiatric Hospital, 18(3),121125,1987 .

CRAIG, C., RAY, F., HIX, C. Seclusion and restraint: Decreasing the discomfort. Journal of Psychosocial Nur sing and Mental Health Services, 27(2), 16-19, 1989.

DARCY, P.T. Theory and Practice of Psychiatric Care. Hodder \& Stoughton, London, 1984.
DE CANGAS, J.,SHOPFLOCHER, D. The practice of seclusion and factors affecting its use. In Chi-Hui (Kao) Lo (Ed.). Proceedings of the Sigma Theta Tau international research congress. Advances in international nursing scholarship. Taipei, Taiwan: Sigma Theta Tau, 83, 1989.

DE CANGAS, J. Nursing staff and unit characteristics: Do they affect the use of seclusion? Perspectives in Psy chiatric Care, 29(3), 15-22, 1993.

DI FABRIO, S. Nurses reaction to restraining patients. American Journal of Nursing, 81, 973-975, 1981.

FASSLER, D.,COTTON, N. A national survey on the use of seclusion in the psychiatric treatment of children. Hospital \& Community Psychiatry, 43, 370-374, 1992.

GENERAL ASSEMBLY OF THE UNITED NATIONS. Universal Declaration of Human Rights Resolution 217 (A) (III). United Nations, New York, 1948.

GERLOCK, A., SOLOMONS, H. Factors associated with the seclusion of psychiatric patients. Perspectives in Care, 21(2), 15-19, 1983.

GREY, S., DIERS, D. The effect of staff stress on patient behaviour. Archives of Psychiatric Nursing, 6(1), 26-34, 1992.

GUNDERSON, J. Defining the therapeutic processes in psychiatric milieus. Psychiatry, 41, 327-335, 1978.

GUTHEIL, T. Observations of the theoretical basis for seclusion of the psychiatric in-patients. American Journal of Psychiatry, 195, 325-328, 1978.

GUTHEIL, T.G., DALY, M. Clinical consideration in seclusion room design. Hospital \& Community Psychia tric, 31(4), 268-270, 1980.

HODGKINSON, P. The use of seclusion. Journal of Medicine, Science and Law, 25(3), 215-222, 1985.

HOPKINS OUTLAW, F.,LOWERY, B.J. An attributional study of seclusion and restraint of psychiatric patients. Archives of Psychiatric Nursing, 8(2), 69-77, (April), 1994.

KENNEDY, B.R.,WILLIAMS, C.A.,PESUT, A.J. Hallucinatory experiences of psychiatric patients in seclusion. Archives of Psychiatric Nursing, 8(3),169-176,(June),1994.

KILGALEN, R.K. The effective use of seclusion. Jour nal of Psychiatric Nursing and Mental Health Services. 15, 22-25, 1977.

LANZA, M., KAYNE, H., HICKS, C., MILNER, J. Nursing staff characteristics related to patient assault. Issues in Mental Health Nursing, 12, 253-265, 1991. 
LION, J. Training for battle: Thoughts on managing aggressive patients. Hospital and Community Psychiatry, 38, 882-884, 1987.

MADIANOS, M. Recent advances in community psychiatry and psychosocial rehabilitation in Greece and the Southern European Countries. International Journal of Social Psychiatry, 40:154-164, 1994.

MADANOS, M, TSIANTIS, J., ZACHARAKIS, C. Changing patterns of mental health care in Greece (19841996) European Psychiatry, 14:462-467, 1995.

MATTSON, M.,SACKS, M. Seclusion:Uses and complications. American Journal of Psychiatry, 135, 12101213, 1978.

McCOY, S.,GARRITSON, S. Seclusion:the process of intervening. Journal of Psychosocial Nursing and Mental Health Services 21 (8), 9- 15, 1983.

MENZIES, I.E.P. The Function of Social Systems as a Defence Against Anxiety- A Report of a Study on the Nursing Service of a General Hospital. Tavistock Institute, London, 1970.

MORRISON, E.F. Toward a better understanding of violence in psychiatric settings: Debunking the myths. Archives of Psychiatric Nursing, 7(6), 328-335, 1993.

MORRISSON, E.F. The tradition of toughness: a study of non professional nursing care in psychiatric settings. IMAGE: Journal of Nursing Scholarship 22(1), 32-38, 1989.

MORRISSON, E.F. A comparison of perceptions of aggression and violence by psychiatric nurses. Internatio nal Journal of Nursing Studies 30(3), 261-268, 1993.

MOUGIA, V.N. The effect of involuntary hospitalization on the clinical symptomatology of psychiatric patients by comparison to voluntary hospitalized patients (Doctoral Dissertation University of Athens, Faculty of Nursing), Athens, 1999.

MUIR-COCHRANE, E.,ADULT, G.D. An investigation into nurses'perceptions of secluding patients on closed psychiatric wards. Journal of Advanced Nursing, 23, 555-563, 1996.

OLDHAM, J., RUSSALOFF, L., PRUSNOFSKY, L. Seclusion: Patterns and milieu. Journal of Nervous and Mental Disease, 171, 154- 165, 1983.

OUTLAW, F., LOWERY, B. Seclusion: The nursing challenge. Journal of Psychosocial Nursing and Mental Health Services, 30(4), 13-17, 1992.

PLATI,CH., LEMONIDOU, CH., PRIAMI,M., BROKALALI, H., MANTAS, J. LANARA, V. Physical Restraints in Hospitalized Patients. Proceedings of the $2^{\text {nd }}$
European Conference on Nursing Administration Turku. Finland September 1995.

PLUTCHIK, R., LARASU, T. Towards a rationale for the seclusion process. Journal of Nervous and Mental Diseases 166(8), 571-579, 1978.

PLUTCHIK, R., KARASU, T.,CONTE, H., SIEGEL, B.,JERRET, T. Toward a rationale for the seclusion process. Journal of Nervous and Mental Disease, 166, 579591, 1978.

RAWLS, J. A Theory of Justice. Oxford University Press, Oxford, 1972.

REDMOND, F.G. The use of seclusion room. Quality Review Bulletin 6(8), 20-23, 1980.

ROPER, J.M.,COUTTS, A.,SATHER, J., \& TAYLOR, R. Restraint and seclusion: A standard and standard care plan. Journal of Psychosocial Nursing \& Mental Health Services, 23, 18-23, 1985.

ROSEN, H.,DI GIACOMO, J.N. The role of the physical restraint in the treatment of psychiatric illness. Journal of Clinical Psychiatry, 39, 228-232, 1978.

ROSENHAN, D.L. On being sane in insane places. Science 179, 250-257, 1973.

ROYAL COLLEGE OF NURSING. Seclusion, Con trol and Restraint. Royal College of Nursing, London, 1992.

SALLAH, D. Points of view. Nursing Standard 6(12), 43, 1992.

SCHWAB, P.J., LAHMEYE, G.B. The uses of seclusion on a general hospital psychiatric unit. Journal of Cli nical Psychiatry 40, 228-231, 1979.

SCLAFANI, M. Violence and behaviour control. Jour nal of Psychosocial Nursing, 24 (11), 8-13, 1986.

SOLOFF, P., TURNER, S.M. Patterns of seclusion: a prospective study. Journal of Nervous and Mental Disor der 169(1), 37-44, 1981.

SOLOFF, P.H. Physical controls: The use of seclusion and restraint in modern psychiatric practice. In L.H. Roth (Ed.) Clinical treatment of the violent person. New York, Guilford, 1987, 119-137.

STILLING, L. The pros and cons of physical restraints and behaviour controls. Journal of Psychosocial Nursing 30(3), 18-20, 1992.

STRUMPF, N., EVANS, L. Physical restraint of the hospitalized elderly. Nursing Research, 37, 132-137, 1988.

TARBUCK, P. Use and abuse of control and restraint. Nursing Standard, 16(6), 30-32, 1992. 
TARDIFF, K.,MATTSON, M. A survey of state mental health directors concerning guidelines for restraint and seclusion. in the Psychiatric Uses of Seclusion and Restraint. Tardiff, K. (Ed.) Washington, DC,American Psychiatric Press, 1984.

THORPE, J.C. Time out or seclusion? Nursing Times, 76(14), 604, 1984.

TOPPING-MORRIS, B. Prisoners of the system. Nur sing Times, 88 (24), 39-41, 1992.
WADESON, H.,CARPENTER, W. Impact of the seclusion room experience. Journal of Nervous and Mental Disease, 163, 318-328, 1976.

Dirección para correspondencia:

C. Lemonidou, Profesor Asociado,

Universidad de Atenas, Escuela de Enfermería, 123 Papadiamantopoulou St.

11527 Atenas,

GRECIA

E-mail: clemonid@cc.uoa.gr 